\title{
2-D periodic structures. A numerical study
}

\author{
Giuseppe Rocchetta
}

Department of Structures for Engineering and Architecture, University of Naples, Naples, Italy, and

Geminiano Mancusi

Department of Civil Engineering, Salerno University, Fisciano, Italy

\begin{abstract}
Purpose - Within the context of 2D square lattices, searching for the existence of band gaps assumes a great interest owing to many possible fields of application: from energy absorption devices to noise and vibration controllers, as well as advanced strategies for the seismic isolation.

Design/methodology/approach - The underlying microstructure may influence the mechanical response of $2 \mathrm{D}$ square lattices according to a complex interplay between different factors. A first one is related to the so-called "size-effect". A second one relates, instead, to the mass density distribution.

Findings - It has been observed that lumped masses may induce additional band gaps to appear and may magnify their width. Finally, an additional factor deals with the inner damping characteristics of the constituent materials, which usually are polymer-based.

Originality/value - This study focuses on the first factor from a specific perspective: to investigate the influence of the size effect on the existence and properties of frequency band gaps.
\end{abstract}

Keywords 2D lattice structures, Frequency band gaps, RUC

Paper type Research paper

\section{Introduction}

The study of the dynamic properties of a 2D lattice (Zhou et al., 2009; Wang et al., 2009; Martinsson and Movchan, 2003; Phani et al., 2006; Zhen et al., 2008; Gaofeng and Zhifei, 2010) is usually based on few assumptions. Firstly, by virtue of the Bloch theorem, the hypothesis of infinite lattice points allows to restrict the study to the representative unit cell (RUC).

An example is shown in Figure 1 , where the symbols $\mathbf{a}_{1}$ and $\mathbf{a}_{2}$ denote the generating vectors along the directions of spatial periodicity. In this example, $\mathbf{a}_{1}$ and $\mathbf{a}_{2}$ are normal to each other.

The displacement of an arbitrary point $\mathrm{P}$ of the infinite $2 \mathrm{D}$ lattice is given by:

$$
\mathbf{u}(\mathbf{r})=\mathbf{u}_{k}(\mathbf{r}) \exp (-i \omega t+\mathbf{k} \cdot \mathbf{r})
$$

(C) Giuseppe Rocchetta and Geminiano Mancusi. Published in PSU Research Review. Published by Emerald Publishing Limited. This article is published under the Creative Commons Attribution (CC BY 4.0) licence. Anyone may reproduce, distribute, translate and create derivative works of this article (for both commercial and non-commercial purposes), subject to full attribution to the original publication and authors. The full terms of this licence may be seen at http://creativecommons.org/ licences/by/4.0/legalcode

G.M. acknowledges financial support from the Italian Ministry of Education, University and Research (MIUR) under the "Departments of Excellence" Grant L.232/2016.

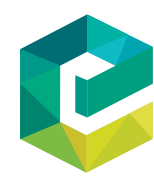

PSU Research Review 
PRR

2,2

176

where the symbol $\mathbf{r}$ indicates the position vector of a generic material point $\mathbf{P}$, the symbol $\mathbf{k}$ denotes the Bloch wave vector, $\omega$ is the angular frequency and $\mathbf{u}_{k}(\mathbf{r})$ is the amplitude. It is important to remark that $\mathbf{u}_{k}(\mathbf{r})$ and the point lattice exhibit the same spatial periodicity. Finally, the position vector $\mathbf{r}$ is expressed by:

$$
\mathbf{r}=\mathbf{r}_{0}+n_{1} \mathbf{a}_{1}+n_{2} \mathbf{a}_{2}
$$

where $\left(n_{1}, n_{2}\right)$ is an integer pair and $\mathbf{r}_{0}$ is the position vector of $\mathbf{O}$, which is the corresponding point of $\mathbf{P}$ within the RUC. Equation (1) becomes:

$$
\mathbf{u}(\mathbf{r})=\mathbf{u}\left(\mathbf{r}_{0}\right) \exp \left(n_{1} \mathbf{k} \cdot \mathbf{a}_{1}+n_{2} \mathbf{k} \cdot \mathbf{a}_{2}\right)
$$

The periodic boundary condition for the dynamic analysis of the unit cell assumes the following final form:

$$
\mathbf{u}(\mathbf{r})=\mathbf{u}\left(\mathbf{r}_{0}\right) \exp \left[2 \pi\left(n_{1} k_{1}+n_{2} k_{2}\right)\right]
$$

Equation (4) is true if the Bloch wave vector is represented by means of a linear combination of the reciprocal space vectors $\mathbf{b}_{1}$ and $\mathbf{b}_{2}$ :

$$
k=k_{1} b_{1}+k_{2} b_{2}
$$

\section{Numerical experimentation and assumptions}

The numerical results presented in this study deal with two RUC configurations: a primary configuration (I) made of four micro-beams [Figure 2(a)] and a different configuration (II) which has been enhanced by means of four auxiliary micro-beams [Figure 2(b)].

We assume that the spatial periodicity vectors $\mathbf{a}_{1}$ and $\mathbf{a}_{2}$ are orthogonal to each other with the same norm $a$. This implies the study is limited to square lattice only. Moreover we assume the RUC configuration can be modelled by interconnecting straight micro-beams with rigid internal connections. Finally, an appropriate micro-scale parameter is introduced to account for the so-called size effect (Mancusi and Feo, 2013; Mancusi et al., 2017; Mindlin, 1963; Lui and Su, 2009; Onck et al., 2000; Andrews et al., 2000; Park and Gao, 2006; Barretta et al., 2017; Ma et al., 2008). The followed approach consists in considering the contribution of couple stresses in addition to classical Cauchy stresses. To this scope, a further constitutive parameter is required. This parameter has with the physical dimension of a scale length (Mindlin, 1963). We want to remark that couple stresses are considered in a

Figure 1.

Two-dimensional square lattice material (example of a RUC)

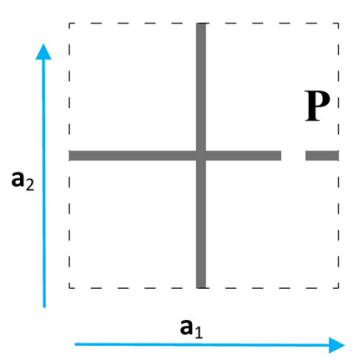


simplified manner, according to the so-called modified couple stress theory, assuming that only the symmetric part of the rotation gradient contributes to the strain energy density.

Geometric and mechanical information of the reference unit cell considered in this study are listed in the following Table I.

The following hypotheses are also taken into account:

- primary micro-beams are composed of aluminium: $\mathrm{E}=9.00 \times 104 \mathrm{~N} / \mathrm{mm}^{2} ; v=0.23$; $\mathrm{G}=3.66 \times 10^{4} \mathrm{~N} / \mathrm{mm}^{2} ; l=6.58 \mu \mathrm{m} ; \rho=2.70 \times 10^{-6} \mathrm{~kg} / \mathrm{mm}^{3}$;

- auxiliary micro-beams are composed of aluminium (i), as above, or epoxy resin (ii): $\mathrm{E}=1440 \mathrm{~N} / \mathrm{mm}^{2} ; v=0.38 ; \mathrm{G}=522 \mathrm{~N} / \mathrm{mm}^{2} ; l=17.6 \mu \mathrm{m} ; \rho=1.10 \times 10^{-6} \mathrm{~kg} / \mathrm{mm}^{3}$.

Numerical results are relative to the following two choices:

(1) the micro-scale characteristic length $l$ is zero (i.e. the size effect is discarded); and

(2) the size effect is accounted for.

It is worth remarking that the present analysis represents a generalization of the parametric analysis discussed by Mancusi et al. (2017).

Ten finite elements over each micro-beams are considered for the numerical analysis. The convergence rate and the accuracy of the numerical solutions have been adequately assessed (Mancusi and Feo, 2013; Mancusi et al., 2017).

The dimensionless frequencies which are detected by means of an eigenvalue problem, are studied over the boundary of the irreducible part of the first Brillouin zone and are presented in a non-dimensional form as $\tilde{\omega}=\omega / \omega_{1}$, where:

$$
\omega_{1}=\frac{\pi^{2}}{a^{2}} \sqrt{\frac{E I}{\rho A}}
$$

(I)

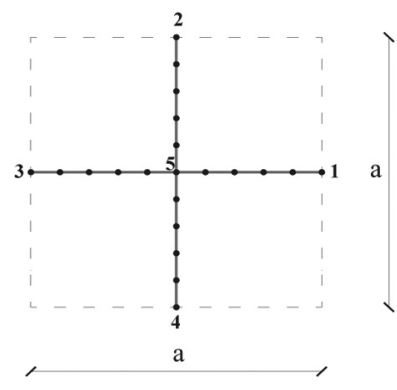

(II)

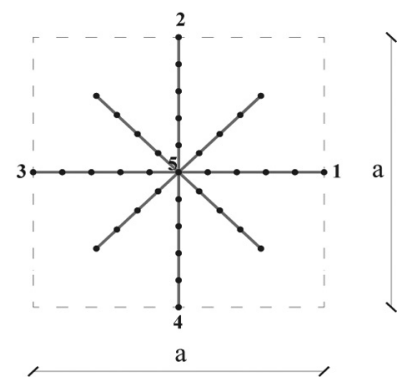

2-D periodic structures

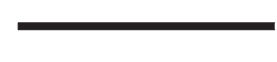


PRR

2,2

178

In Table II, the main results concerning the lowest natural frequencies (not dimensional) are presented. They refer to the points $\mathbf{O}, \mathbf{A}$ and $\mathbf{B}$ indicated in Figure 3.

A comparison emerges between two different hypotheses concerning the constituent material of the auxiliary micro-beams:

(1) aluminium (i.e. the same material as the primary micro-beams); or

(2) epoxy resin.

Moreover, the influence of the microstructure length is also considered according to the assumptions previously summarized by acronyms "a" and "b".

In Table III, the comparison is finally extended to the position and the width of the first band gaps. The row indicates as "lower band" denotes the number of the frequency band immediately below the considered gap.

\section{Final remarks}

It has been observed that the position and width of the first band gaps are influenced by the magnitude of the micro-scale parameter $l$. In general, a forward shift of the first band gaps is related to the size effect. Moreover, flat bands also appear in the low-frequency region if auxiliary micro-beams are present. Finally a not-uniform mass density distribution over the RUC may influence the band structure of the $2 \mathrm{D}$ periodic material.

\begin{tabular}{|c|c|c|c|c|c|c|c|c|c|c|c|}
\hline Points & & 1 & 2 & 3 & 4 & 5 & 6 & 7 & 8 & 9 & 10 \\
\hline \multirow[t]{3}{*}{ 1.a } & $\mathrm{O}$ & 0.0 & 0.0 & 4.2 & 4.2 & 6.1 & 9.3 & 18.8 & 18.8 & 23.9 & 29.4 \\
\hline & A & 0.8 & 2.4 & 3.3 & 7.0 & 11.5 & 13.2 & 14.5 & 20.0 & 25.5 & 32.9 \\
\hline & $\mathrm{B}$ & 1.7 & 2.2 & 8.9 & 11.6 & 11.6 & 12.7 & 17.0 & 17.0 & 28.9 & 34.7 \\
\hline \multirow[t]{3}{*}{ 1.b } & $\mathrm{O}$ & 0.0 & 0.0 & 10.8 & 10.8 & 15.9 & 21.7 & 37.1 & 37.1 & 52.1 & 56.4 \\
\hline & A & 2.0 & 6.6 & 7.5 & 15.9 & 17.8 & 25.7 & 30.4 & 36.6 & 51.6 & 53.2 \\
\hline & B & 4.2 & 5.7 & 14.1 & 14.1 & 22.1 & 27.4 & 30.6 & 30.9 & 48.2 & 48.3 \\
\hline \multirow[t]{3}{*}{ 2.a.i } & $\mathrm{O}$ & 0.0 & 0.0 & 2.6 & 3.7 & 4.0 & 4.3 & 4.3 & 4.8 & 7.9 & 9.3 \\
\hline & A & 0.6 & 2.2 & 3.0 & 3.3 & 4.2 & 4.3 & 4.3 & 8.4 & 10.3 & 11.9 \\
\hline & B & 1.5 & 2.2 & 3.0 & 4.2 & 4.3 & 4.3 & 9.7 & 9.8 & 10.7 & 12.7 \\
\hline \multirow[t]{3}{*}{ 2.a.ii } & $\mathrm{O}$ & 0.0 & 0.0 & 1.1 & 1.1 & 1.1 & 1.1 & 4.0 & 4.0 & 5.8 & 6.3 \\
\hline & A & 0.6 & 1.1 & 1.1 & 1.1 & 1.2 & 2.3 & 3.3 & 6.2 & 6.3 & 6.3 \\
\hline & $\mathrm{B}$ & 1.0 & 1.1 & 1.1 & 1.1 & 1.7 & 2.2 & 6.1 & 6.3 & 6.3 & 6.3 \\
\hline \multirow[t]{3}{*}{ 2.b.i } & $\mathrm{O}$ & 0.0 & 0.0 & 7.0 & 9.6 & 10.2 & 11.4 & 11.4 & 12.7 & 19.5 & 21.7 \\
\hline & A & 1.5 & 6.0 & 6.8 & 8.0 & 10.1 & 11.4 & 11.4 & 14.5 & 20.4 & 26.4 \\
\hline & B & 3.7 & 5.7 & 7.7 & 8.8 & 11.4 & 11.4 & 11.5 & 13.8 & 25.0 & 27.3 \\
\hline \multirow[t]{3}{*}{ 2.b.ii } & $\mathrm{O}$ & 0.0 & 0.0 & 1.2 & 1.2 & 1.2 & 1.2 & 6.9 & 6.9 & 6.9 & 7 \\
\hline & A & 1.2 & 1.2 & 1.2 & 1.2 & 2.0 & 6.2 & 6.8 & 6.9 & 6.9 & 7.1 \\
\hline & $\mathrm{B}$ & 1.2 & 1.2 & 1.2 & 1.2 & 4.2 & 5.7 & 6.9 & 6.9 & 6.9 & 6.9 \\
\hline
\end{tabular}

Figure 3.

Irreducible part of the first Brillouin zone

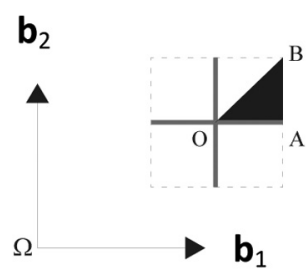




\begin{tabular}{|c|c|c|c|c|c|c|c|c|c|c|c|c|}
\hline & & 1 & 2 & 3 & 4 & 5 & 6 & 7 & 8 & 9 & 10 & \\
\hline Position & 1.a & 3.300 & 13.860 & 21.936 & 29.166 & 35.260 & 45.858 & 66.796 & 74.461 & 89.245 & 105.955 & \\
\hline Gap & & 0.046 & 1.347 & 3.856 & 0.472 & 1.133 & 7.388 & 1.283 & 0.672 & 5.260 & 0.910 & \\
\hline Lower band & & 2 & 6 & 8 & 9 & 10 & 12 & 16 & 18 & 20 & 22 & \\
\hline Position & 1.b & 7.263 & 15.914 & 28.868 & 42.659 & 64.808 & 74.250 & 91.520 & 104.320 & 118.630 & 156.655 & \\
\hline Gap & & 0.280 & 0.044 & 2.999 & 11.055 & 5.240 & 3.774 & 7.614 & 8.200 & 8.280 & 10.170 & 17 \\
\hline Lower band & & 2 & 4 & 6 & 8 & 12 & 14 & 16 & 18 & 20 & 26 & \\
\hline Position & 2.a.i & 3.184 & 13.449 & 20.105 & 24.745 & 26.916 & 28.006 & 35.162 & 45.711 & 51.342 & 67.185 & \\
\hline Gap & & 0.115 & 1.439 & 2.704 & 3.840 & 0.501 & 1.205 & 0.942 & 3.948 & 7.314 & 0.505 & \\
\hline Lower band & & 3 & 10 & 12 & 13 & 15 & 16 & 18 & 22 & 24 & 28 & \\
\hline Position & 2.a.ii & 1.068 & 1.075 & 3.240 & 14.644 & 16.943 & 22.136 & 29.207 & 35.240 & 44.295 & 46.572 & \\
\hline Gap & & 0.001 & 0.013 & 0.061 & 0.213 & 0.012 & 3.828 & 0.390 & 1.094 & 4.155 & 0.049 & \\
\hline Lower band & & 2 & 4 & 6 & 18 & 22 & 24 & 25 & 30 & 36 & 38 & \\
\hline Position & 2.b.i & 6.551 & 7.743 & 12.121 & 17.030 & 28.808 & 36.918 & 44.041 & 47.837 & 49.800 & 51.654 & \\
\hline Gap & & 0.139 & 0.085 & 1.228 & 5.022 & 3.019 & 0.571 & 7.288 & 0.303 & 2.460 & 1.002 & \\
\hline Lower band & & 2 & 3 & 7 & 8 & 10 & 12 & 14 & 16 & 17 & 18 & \\
\hline Position & 2.b.ii & 1.205 & 1.225 & 6.639 & 7.299 & 11.587 & 12.471 & 28.859 & 36.909 & 42.145 & 47.197 & Table III. \\
\hline Gap & & 0.001 & 0.040 & 0.115 & 0.166 & 0.843 & 0.925 & 2.992 & 0.157 & 9.421 & 0.053 & Position and width \\
\hline Lower band & & 2 & 4 & 6 & 10 & 12 & 14 & 22 & 28 & 32 & 34 & for the lowest gaps \\
\hline
\end{tabular}

\section{References}

Andrews, E.W., Gioux, G., Onck, P.R. and Gibson, L.J. (2000), "Size effects in ductile cellular solids. part II: experimental results”, International Journal of Mechanical Sciences, Vol. 43 No. 3, pp. 701-713.

Barretta, R., Feo, L., Luciano, R., Marotti de Sciarra, F. and Penna, R. (2017), "Nano-beams under torsion: a stress-driven nonlocal approach”, PSU Research Review, Vol. 1 No. 2, pp. 164-169.

Gaofeng, J. and Zhifei, S. (2010), "A new seismic isolation system and its feasibility study", Earthquake Engineering and Engineering Vibration, Vol. 9 No. 1, pp. 75-82.

Lui, S. and Su, W. (2009), "Effective couple-stress continuum model of cellular solids and size effects analysis", International Journal of Solids and Structures, Vol. 46 Nos 14/15, pp. 2787-2799.

Ma, H.M., Gao, X.L. and Reddy, J.N. (2008), “A microstructure-dependent timoshenko beam model based on a modified couple stress theory", Journal of the Mechanics and Physics of Solids, Vol. 56 No. 12, pp. 3379-3391.

Mancusi, G. and Feo, L. (2013), "A refined finite element formulation for the Microstructure-Dependent analysis of Two-Dimensional (2D) lattice materials", Materials, Vol. 6 No. 1, pp. 1-17.

Mancusi, G., Fabbrocino, F., Feo, L. and Fraternali, F. (2017), "Size effect and dynamic properties of 2D lattice materials", Composites Part B, Vol. 112, pp. 235-242.

Martinsson, P.G. and Movchan, A.B. (2003), "Vibrations of lattice structures and phononic band gaps", The Quarterly Journal of Mechanics and Applied Mathematics, Vol. 56 No. 1, pp. 45-64.

Mindlin, R.D. (1963), "Influence of couple-stresses on stress concentrations", Experimental Mechanics, Vol. 3 No. 1, pp. 1-7.

Onck, P.R., Andrews, E.W. and Gibson, L.J. (2000), "Size effects in ductile cellular solids. Part I: modelling”, International Journal of Mechanical Sciences, Vol. 43 No. 3, pp. 681-699.

Park, S.K. and Gao, X.L. (2006), "Bernoulli-Euler beam model based on a modified couple stress theory", Journal of Micromechanics and Microengineering, Vol. 16 No. 11, pp. 2355-2359.

Phani, A.S., Woodhouse, J. and Fleck, N.A. (2006), "Wave propagation in two-dimensional periodic lattices", The Journal of the Acoustical Society of America, Vol. 119 No. 4, pp. 1995-2005. 
PRR

2,2
Wang, Y., Li, F., Wang, Y., Kishimoto, K. and Huang, W. (2009), "Tuning of band gaps for a twodimensional piezoelectric phononic crystal with a rectangular lattice", Acta Mechanica Sinica, Vol. 25 No. 1, pp. 65-71.

Zhen, N., Yan, Z. and Wang, Y. (2008), "Elastic wave propagation in honeycomb materials", Chinese Journal of Theoretical and Applied Mechanics, Vol. 40, pp. 769-775.

Zhou, X.Z., Wang, Y.S. and Zhang, C.Z. (2009), "Effects of material parameters on elastic band gaps of two-dimensional solid phononic crystals”, Journal of Applied Physics, Vol. 106 No. 1, pp. 14903-14911.

\section{Corresponding author}

Geminiano Mancusi can be contacted at: g.mancusi@unisa.it

For instructions on how to order reprints of this article, please visit our website: 